J. Dairy Sci. 103:4892-4893

https://doi.org/10.3168/jds.2020-103-5-4892

(๑) American Dairy Science Association ${ }^{\circledR}, 2020$.

\title{
Corrigendum to: "Short communication: Electrochemical activated drinking water effects on bovine milk production and composition, including chlorate, perchlorate, and fatty acid profile" (J. Dairy Sci. 103:1208-1214)
}

Einar Vargas-Bello-Pérez, Rajan Dhakal, Mette O. Nielsen, Lilia Ahrné, and Hanne H. Hansen

Units of measurement for chlorate and perchlorate in Table 1 (page 1211) and Table 4 (page 1213) were incorrect and should be modified as shown below (corrected units in bold). In Table 1, values were shown as $\mu \mathrm{g} / \mathrm{kg}$, but should be $\mathrm{mg} / \mathrm{kg}$; in Table 4 , they were shown as $\mathrm{mg} / \mathrm{L}$ but should be $\mu \mathrm{g} / \mathrm{L}$.

On page 1212, the second sentence of the second column should be corrected as follows: "It is important to note that the maximum residue limit allowed for chlorate in EC listed foods [regulation (EC) no. 396/2005] is 0.01 mg/kg (EFSA, 2015)."

Although the European Food Safety Association (EFSA) has not established a maximum level for chlorate in water, the World Health Organization has established a guideline maximum level of $0.7 \mathrm{mg} / \mathrm{L}$ in drinking water.

The authors regret the errors.

\section{REFERENCES}

Vargas-Bello-Perez, E., R. Dhakal, M. O. Nielsen, L. Ahrne, and H. H. Hansen.. 2020. Short communication: Effects of electrochemically activated drinking water on bovine milk production and composition, including chlorate, perchlorate, and fatty acid profile. J. Dairy Sci. 103(2):1208-1214. https://doi.org/10.3168/jds.2019-17510.

Table 1. Milk production, milk composition, and milk metabolites from cows fed with or without electrochemically activated water (ECW; $\mathrm{n}$ $=5$ cows per treatment unless otherwise stated $)^{1}$

\begin{tabular}{|c|c|c|c|c|c|c|}
\hline \multirow[b]{2}{*}{ Parameter } & \multicolumn{2}{|c|}{ Treatment } & \multirow[b]{2}{*}{ SEM } & \multicolumn{3}{|c|}{$P$-value } \\
\hline & Control & ECW & & $\begin{array}{l}\text { Treatment } \\
\quad(\mathrm{T})\end{array}$ & $\begin{array}{l}\text { Period } \\
(\mathrm{P})\end{array}$ & $\mathrm{T} \times \mathrm{P}$ \\
\hline Milk yield, $\mathrm{kg}$ of ECM (61 cows per treatment) & 32.6 & 33.4 & 0.87 & NS & $<0.001$ & $<0.001$ \\
\hline Milk yield, $\mathrm{kg}$ of ECM & 30.4 & 32.7 & 1.50 & NS & $<0.001$ & NS \\
\hline \multicolumn{7}{|l|}{ Milk composition } \\
\hline SCC,$\times 10^{3} / \mathrm{mL}(61$ cows per treatment $)$ & 311.78 & 243.59 & 49.98 & NS & NS & 0.026 \\
\hline Lactose, $\%$ & 4.51 & 4.46 & 0.05 & NS & NS & NS \\
\hline Protein, $\%$ & 4.06 & 3.88 & 0.07 & NS & 0.06 & NS \\
\hline MUN, mmol/L & 3.95 & 4.59 & 0.17 & 0.02 & $<0.001$ & $<0.001$ \\
\hline \multicolumn{7}{|l|}{ Milk metabolite } \\
\hline Acetone, mmol/L & 0.06 & 0.05 & 0.01 & NS & $<0.001$ & 0.083 \\
\hline $\mathrm{BHB}, \mathrm{mmol} / \mathrm{L}$ & 0.11 & 0.04 & 0.02 & 0.03 & 0.01 & NS \\
\hline Iodine, $\mu \mathrm{g} / \mathrm{kg}$ & 173.10 & 178.40 & 22.04 & NS & $<0.001$ & NS \\
\hline
\end{tabular}

${ }^{1}$ The ECM was calculated according to the Nordic feed evaluation system (NorFor; Volden, 2011). 
Table 4. Chlorate and perchlorate contents in different sites from the water supply of the farm

\begin{tabular}{lccc}
\hline Location & $\begin{array}{c}\text { Period, } \\
\mathrm{d}\end{array}$ & $\begin{array}{c}\text { Chlorate, } \\
\boldsymbol{\mu g} / \mathbf{L}\end{array}$ & $\begin{array}{c}\text { Perchlorate, } \\
\boldsymbol{\mu g} / \mathbf{L}\end{array}$ \\
\hline Pipeline without ECW & $<2$ & $<0.5$ \\
Pipeline with ECW & & $<2$ & $<0.5$ \\
Tap water & 0 & $<2$ & $<0.5$ \\
Drinking troughs without ECW & & $<2$ & $<0.5$ \\
Drinking troughs with ECW & & $<2$ & $<0.5$ \\
Pipeline without ECW & & $<2$ & $<0.5$ \\
Pipeline with ECW & 30 & 190 & $<0.5$ \\
Tap water & & $<2$ & $<0.5$ \\
Drinking troughs without ECW & & $<2$ & $<0.5$ \\
Drinking troughs with ECW & 60 & 280 & $<0.5$ \\
Pipeline without ECW & & $<2$ & $<0.5$ \\
Pipeline with ECW & & 490 & $<0.5$ \\
Tap water & & $<2$ & $<0.5$ \\
Drinking troughs without ECW & 330 & $<0.5$ \\
Drinking troughs with ECW & $<2$ & $<0.5$ \\
Pipeline without ECW & $<0.5$ \\
Pipeline with ECW & & $<2$ & $<0.5$ \\
Tap water & & $<2$ & $<0.5$ \\
Drinking troughs without ECW & $<2$ & $<2$ \\
Drinking troughs with ECW & & $<2$ & $<2$ \\
\hline
\end{tabular}

${ }^{1} \mathrm{ECW}=$ electrochemically activated water. 